\title{
TRANSPLANTATION OF CRICKET SENSORY NEURONS TO ECTOPIC LOCATIONS: ARBORIZATIONS AND SYNAPTIC CONNECTIONS ${ }^{1}$
}

\author{
R. K. MURPHEY, ${ }^{2}$ J. P. BACON, D. S. SAKAGUCHI, AND S. E. JOHNSON \\ Neurobiology Research Center, Department of Biological Sciences, State University of New York at Albany, \\ Albany, New York 12222
}

Received September 8, 1982; Revised November 22, 1982; Accepted November 23, 1982

\begin{abstract}
The cerci (abdominal sensory appendages) of crickets were transplanted to a leg stump after amputating the leg. Single identifiable cercal afferents were stained and found to regenerate into the host thoracic ganglia. A given neuron always arborizes in the same area of neuropil of the foreign ganglion and is distinctive in this property from other identified neurons. Taken as a whole, the results show that the afferents from the ectopic cercus are spatially ordered, the destination of a particular afferent within the ganglion being correlated with the location of its sensory cell body on the cercal surface. This is the case for the pro-, meso-, and metathoracic ganglion and the topography of these ectopic projections bears some resemblance to the normal projections found in the terminal ganglion. Thus the insect segmental ganglion seems to possess a set of markers which are interpretable by all afferent neurons, and this organization is repeated in each ganglion.

The ectopic afferents make functional synaptic connections with intersegmental interneurons, one of which is described anatomically here. However, the ectopic afferents do not, as had previously been reported, make functional connections with the medial giant or lateral giant interneuron (the normal targets of cercal sensory neurons in the terminal ganglion).
\end{abstract}

The arthropod nervous system is convenient for testing hypotheses concerning neuronal specificity and pathfinding ability. In these animals, sensory-neurons are derived from the epidermis and their somata are located in the epidermis. Because arthropods have no immune system, it is relatively easy to transplant a piece of epidermis, containing sensory neurons, from one specimen to another of the same species or from one location to another within an animal. In this way, the growth of neurons from an altered peripheral location into foreign target areas of the central nervous system (CNS) can be studied.

It has long been thought that the basic unit of the invertebrate CNS is the segmentally derived ganglion. Within each ganglion, a rind of somata surrounds a neuropil made up of the dendrites of interneurons and motor neurons and the terminal arborizations of sensory neurons. The neuropil of these ganglia is divided into regions subserving different areas of the body, different modalities, and different functions, and it has recently

\footnotetext{
'We thank Bill Walthall and Gwen Jacobs for editorial assistance. Linda Welch, Ryland Loos, and Bob Speck helped prepare the manuscript for publication. Supported by National Science Foundation Grant BNS81-19799 and National Institutes of Health Grant NS15571.

${ }^{2}$ To whom corresponsence should be addressed.
}

been suggested that within these regions the afferents are somatotopically ordered. For example, mechanoreceptive afferents on the cercus of crickets project to a special region of the terminal abdominal ganglion called the cercal glomerulus, and within this region of neuropil the terminal arborizations of sensory neurons are arranged in a way which topologically parallels their position on the cercal surface, thereby producing a somatotopic map (Murphey et al., 1980; Murphey, 1981).

We have used our knowledge of the cricket cercal sensory system lo study two questions. First, is the orderly organization of the cercal glomerulus a general feature of ganglionic organization throughout the animal? We reasoned that by transplanting the cercus to ectopic sites in the thorax, we could use the regenerating cercal sensory neurons as probes for the orderliness of the neuropil of the foreign ganglion. Our results demonstrate that many aspects of the cercal afferent projection are retained when the sensory neurons arborize in any of the three thoracic ganglia. Thus it appears that the orderliness is segmentally repeated and that the cues used by ingrowing sensory neurons can be interpreted wherever they are encountered. Second, these experiments provided an opportunity to ask how specific are the synaptic connections made by ectopic sensory neu- 
rons? It has been suggested that cercal afferents, when transplanted to thoracic sites, seek out and synapse with their normal targets, the abdominal giant neurons, as they pass through the thorax (Edwards and Sahota, 1967). We reexamined this idea with intracellular techniques. Although both the ectopic afferents and the abdominal giant neurons were found to arborize in the thoracic ganglia, we found no evidence for functional connections between the afferents and these interneurons. This contrasts sharply with the interpretation of Edwards and Sahota (1967). The ectopic afferents did, however, make functional connections with other interneurons, and we presume it is these foreign target interneurons that Edwards and Sahota (1967) recorded in their experiments.

\section{Materials and Methods}

\section{Surgery}

Specimens were produced for these experiments by transplanting a cercus from the abdomen of one cricket, Acheta domesticus, to the thorax of another (Figs. 1 and $2 A$ ). The leg of the host specimen was amputated in the middle of the coxa and a cercus from a sister specimen of the same age was inserted into the stump and held in place until the hemolymph coagulated. The handedness of the cercus and leg were held constant; for example, a right cercus would be implanted into a right leg stump, but never into a left. The cercus was aligned in the leg so that the leg coordinates and cercal coordinates were matched as nearly as possible. We have produced approximately 500 specimens in this way with a surgical success rate of approximately $30 \%$. Only a portion of these had an ectopic cercus which was suitable for anatomical or physiological work and the results presented here are based on approximately 100 useful animals.

\section{Scanning electron microscopy (SEM)}

Animals were prepared for SEM by fixation in Carnoy's fixative followed by dehydration in acetone. They were then critical point dried, glued to studs with silver paint, and sputter coated with gold. Specimens were viewed and photographed on an ETEC Autoscan microscope.

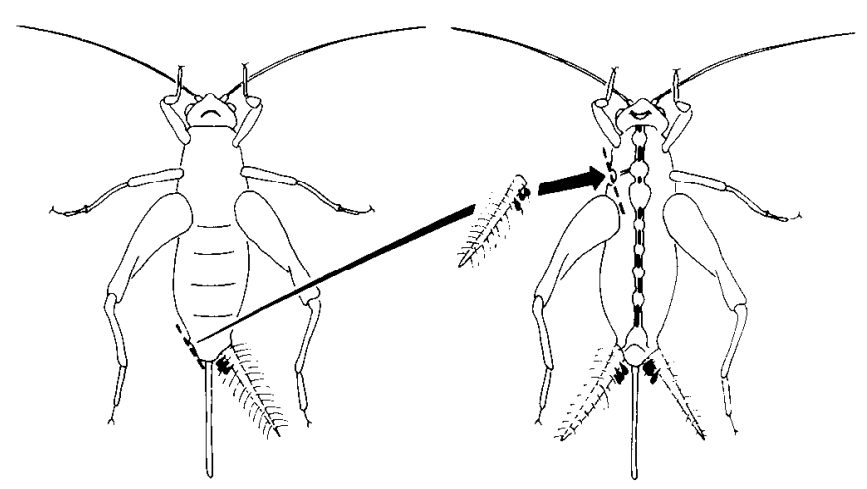

Figure 1. Diagram showing the operation used to produce the experimental specimens used throughout the paper. The transplant is performed between specimens. The dorsal-ventral axes of the transplant are matched to those of the host coxa at the time of the operation.

\section{Staining of afferents}

The methods for staining afferents in the cercal system have been described in previous publications (Murphey et al., 1980; Murphey, 1981). The only addition we have made is to embed the preparations in soft Mollenhauer's Epon/Araldite (Mollenhauer, 1964; mixture 2 modified using up to $20 \%$ less dodecenyl succinic anhydride) before cutting thick sections (20 to $40 \mu \mathrm{m}$ ) with a steel knife. Where necessary, the sections were counterstained with $1 \%$ toluidine blue in order to observe internal ganglionic structures which were not stained with cobalt.

\section{Physiology}

The methods used have been described elsewhere (Matsumoto and Murphey, 1977; Murphey et al., 1977). The only modification of these standard techniques is the use of $100 \mathrm{~mm}$ hexaminecobaltic chloride solutions for the intracellular iontophoretic staining of neurons (Brogan and Pitman, 1981). This substance produces electrodes which are adequate for physiology and which seldom block during the passage of dye. This dramatically improved our success rate of staining neurons (compared with cobalt chloride), especially when fine electrodes were used to explore the connectives.

\section{Results}

\section{Structure of ectopic cerci}

Normal cerci possess a specialized group of mechanoreceptors on their medial aspect called clavate hairs. Cerci were transplanted to the coxa with the clavate hairs positioned posteriorly on the leg stump. It is known that minor misalignments of the host and transplant coordinates are corrected by physical rotation of the graft during healing, whereas major misalignments are not corrected and often induce the production of supernumary tissue (French et al., 1976). The final orientation of our successful grafts, with respect to the host tissue, suggests that the medial aspect of the cercus corresponds to the posterior aspect of the leg.

Individual clavate hairs are identifiable in each animal of the species (Murphey et al., 1980; Murphey, 1981) and the ectopic cerci possess a full complement of these identifiable hairs (Fig. $2 B$ ). In this paper we will focus our attention on five clavate receptors numbered 10,20 , 40,60 , and 80 , which represent three positions on the cercus; ventromedial (hairs 10 and 20), medial (hairs 40 and 60), and dorsomedial (hair 80) (Fig. $2 B$ and Fig. 5, inset).

A second class of mechanoreceptors, known as filiform hairs, is also present on these ectopic cerci. As on the normal cercus, filiform hairs situated dorsally and ventrally (cercal coordinates) on the ectopic cercus vibrate transversely to the long axis of the cercus ( $\mathrm{T}$-hairs), while those situated medially and laterally vibrate in a plane parallel to the longitudinal axis of the cercus (L-hairs). Many of these hairs are identifiable in young specimens, but on adult cerci it is difficult to reliably identify individuals because of the large numbers of hairs. Since we were unable to identify individual filiform hairs on the ectopic cercus, we defined hairs in terms of their position on the cercus and their preferred plane of vibration ( $\mathrm{T}$ versus $\mathrm{L}$ ). For the purpose of this study, we chose $\mathrm{T}$ - 

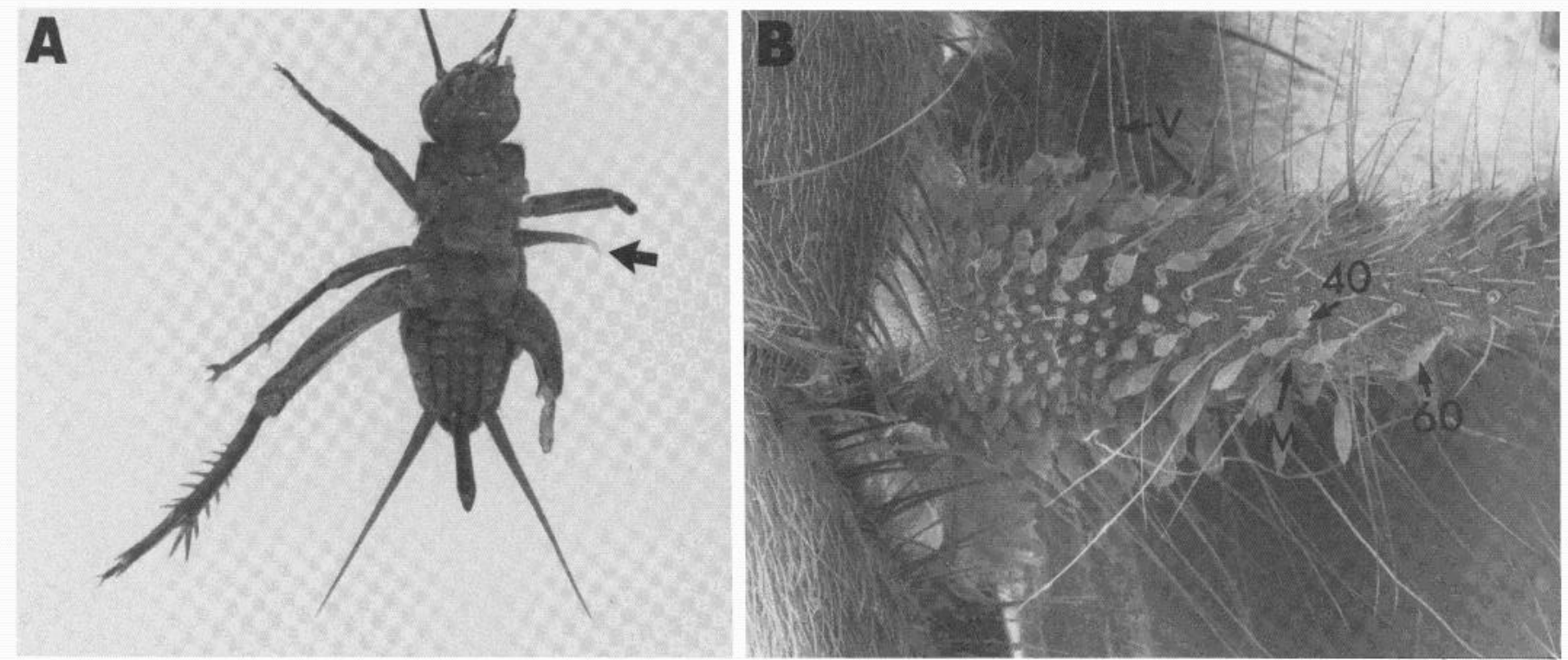

Figure 2. The location and structure of the ectopic cercus. A, Light micrograph of an experimental specimen shown in ventral view. The arrow indicates the ectopic cercus. The pro- and metathoracic legs were amputated at the tibia to prevent the animal from grooming the ectopic cercus. $B$, SEM of the proximal medial aspect of the cercus. Note the presence of a normally organized clavate receptor array. Clavate hairs 40 and 60 are labeled as are a ventral T-hair $(V)$ and a medial L-hair $(M)$.
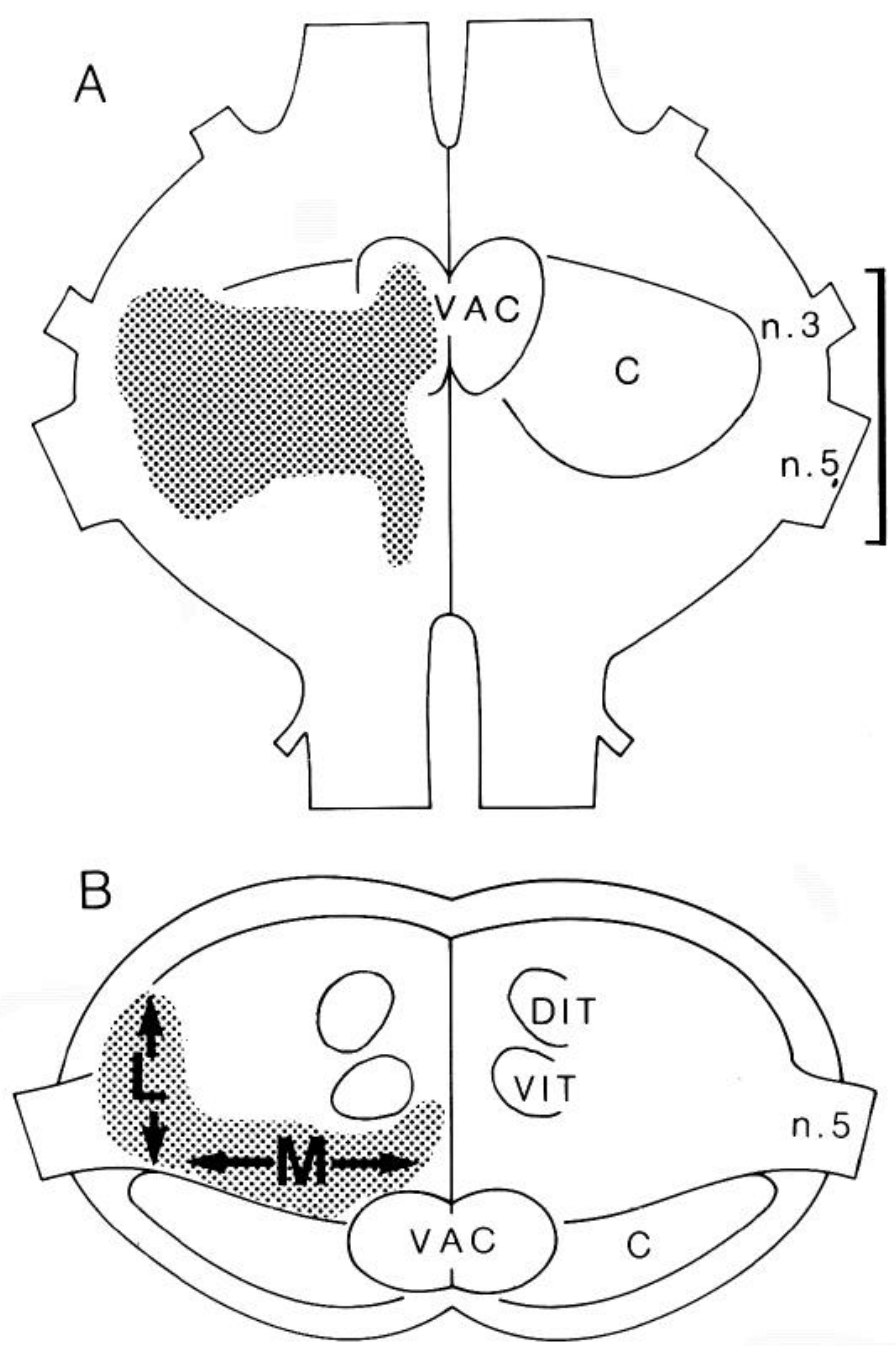

hairs in two positions, dorsal hairs near clavate row 90 and ventral hairs near clavate hair 10 (labeled $V$ in Fig. $2 B$ ). Two types of L-hair were also chosen: a medial group comprised of filiform hairs within the clavate array (labeled $M$ in Fig. $2 B$ ) and a lateral group located opposite the medial hairs on the lateral aspect of the ectopic cercus.

The fact that individual clavate hairs or groups of $\mathrm{T}$ or $\mathrm{L}$ filiform hairs could be identified as readily on the ectopic as on the normal cercus demonstrates that ectopic cerci have a normal external appearance.

\section{The projection of ectopic clavate hairs}

Mesothoracic ganglion. The overall projection region of the ectopic cercus was revealed by damaging a number

Figure 3 . The area of neuropil occupied by ectopic clavate and filiform terminal arborizations shown in horizontal $(A)$ and transverse $(B)$ view. The total projection was obtained by filling the whole cercal nerve as in Figure $4 A$. The target area of the clavate and filiform hair afferents (stippled area) was determined directly by filling single afferents as in Figure $4, B, C$, and $D$. In this paper, we have divided the area into a lateral $(L)$ and medial $(M)$ region to facilitate the description of the individual afferent neurons. The target area of the appressed hairs, labeled $C$, was defined in another series of experiments (R. K. Murphey, unpublished experiment). The bracket to the right of $A$ indicates that portion of ganglion portrayed transversely in $B . V A C$, ventral association center, a well defined bilaterally symmetric region of dense ventral neuropil located at the anterior end of the ganglion. $C$, a similarly dense neuropil which extends much farther laterally and is located on the ventral surface of the neuropil. This region has no consistent name in the literature. DIT, dorsal intermediate tract, a well defined group of axons running through all thoracic ganglia. $V I T$, ventral intermediate tract. $n .3$ and $n .5$, peripheral nerves 3 and 5 . 

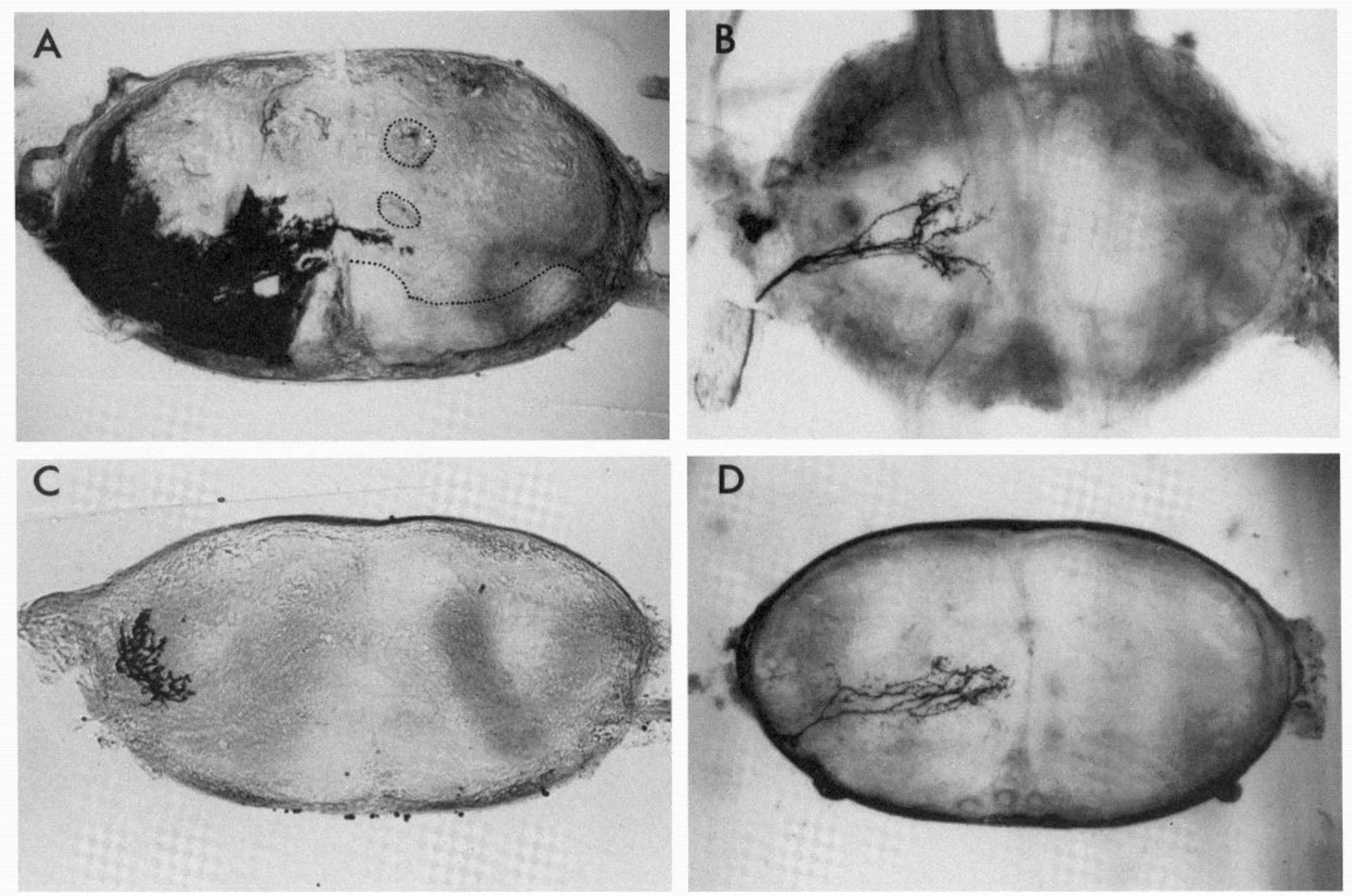

Figure 4. Photomicrographs of ectopic arborizations in the mesothoracic ganglion. $A$, Cross-section of a ganglion in which the entire ectopic arborization was stained. The landmarks used throughout the text are indicated by dotted lines on the right side of the ganglion and are explained in Figure 3. B, Dorsal view of the terminal arborization of clavate neuron 60 in whole mount. $C$, Cross-section through the terminal arborization of a neuron innervating a dorsal T-hair. $D$, Cross-section through the terminal arborization of the neuron innervating a medial L-hair. In dorsal view this neuron is very similar to the one seen in whole mount in $B$.

of receptors at various positions and placing a drop of cobalt chloride solution over the receptors. Viewed dorsally, the area is rectangular with its medial side on the midline (Fig. $3 A$ ). Viewed in transverse section, there are at least two distinct areas of termination: a very ventral region coextensive with the ventral association center (VAC) (Fig. 4A) and a region dorsal to this and ventral to the ventral intermediate tract (VIT) nearer the middle of the ganglion (Figs. $3 B$ and $4 A$ ) (see Pipa and Cook, 1959; Tyrer and Gregory, 1982, for an explanation of this nomenclature). The most ventral slab of neuropil (labeled $C$ in Fig. $3 B$ ) is the target area for the neurons associated with the appressed hairs (R. K. Murphey, unpublished observation). The stippled area just dorsal to this is the target area for the clavate and filiform hair afferents (Fig. $3 B$ ). This area of thoracic neuropil, innervated by the ectopic cercal mechanoreceptors, is the region normally innervated by some leg afferents (Eibl and Huber, 1979; Pflüger et al., 1981).

A given ectopic clavate neuron terminates in characteristic areas of this mesothoracic mechanosensory neuropil in each experimental specimen. Neurons 10 and 80 arborize primarily at the lateral edge of the ganglion, whereas neurons 40 and 60 , located medially on the cercus, arborize more medially in mechanosensory neuropil (Fig. 5, $A$ and $B$ ). The arborizations for both ectopic clavate and filiform hair afferents are described in this paper as being predominantly medial or lateral in the neuropil. This demarcation of the mechanosensory neuropil is shown diagrammatically in Figure $3 B$. These results were highly reproducible, thus demonstrating the correlation between the destination of afferent arborizations within the mesothoracic neuropil and the positions of their sensory cell bodies on the ectopic cercus.

An important feature of the normal arborization within the terminal abdominal ganglion is the continuous nature of the mapping between circumferential position of the neuron on the cercus and the location of its dendritic arborization in the cercal glomerulus (Murphey et al., 1980; Murphey, 1981). In order to search for graded changes in the topography of the ectopic arbors as a function of cercal position, we superimposed all ectopic neurons of a given identity on a single ganglion outline, normalizing for ganglion size which varies slightly from specimen to specimen. The results demonstrate a continuous change in the destination of the terminal arboriza- 

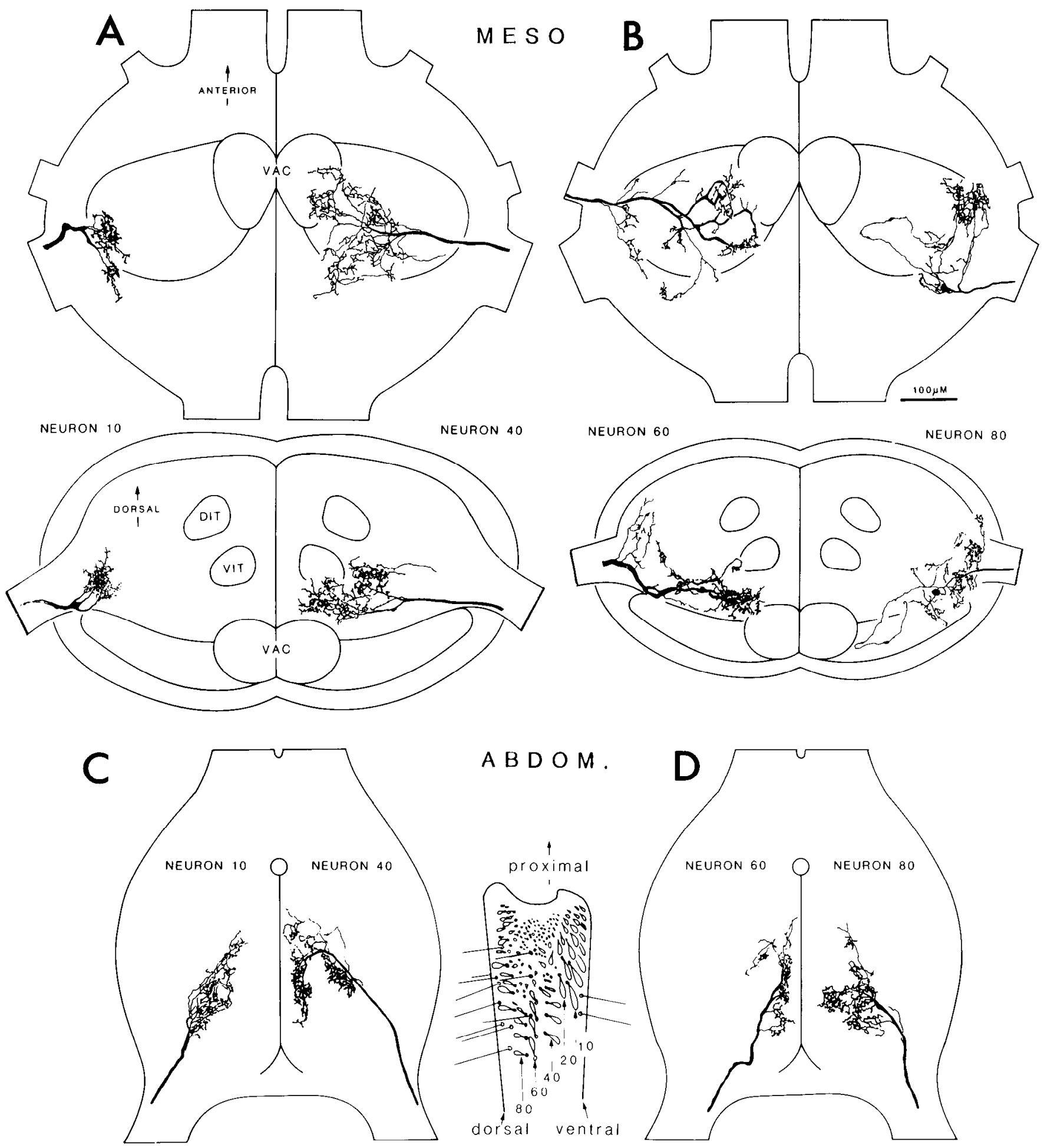

Figure 5. The mesothoracic terminal arborizations of sensory neurons innervating clavate hairs on ectopic cerci. $A$ and $B$, Upper panels: dorsal views of the terminal arborizations; lower panels: transverse views of the same arbors reconstructed from $40-\mu \mathrm{m}$ plastic sections. $C$ and $D$, Dorsal views of the arborizations in the terminal abdominal ganglia. The ganglia outlines are standardized and each arbor is aligned with respect to the indicated landmarks. Inset, Miedial surface of the base of a left cercus showing all of the clavate hairs as well as some of the filiform hairs discussed in the iext. See Figure 3 for an explanation of abbreviations.

tion as a function of position of the sensory neuron soma on the ectopic cercus (Fig. 6). There seem to be two main regions of suitable target area within the ganglion, one more medial than the other, and the gradations we see are gradations in the degree of arborization in one or both regions: neurons 20 and 40 arborize in both regions, whereas neurons 10 and 60 arborize primarily in one or the other. These results strongly support the contention 

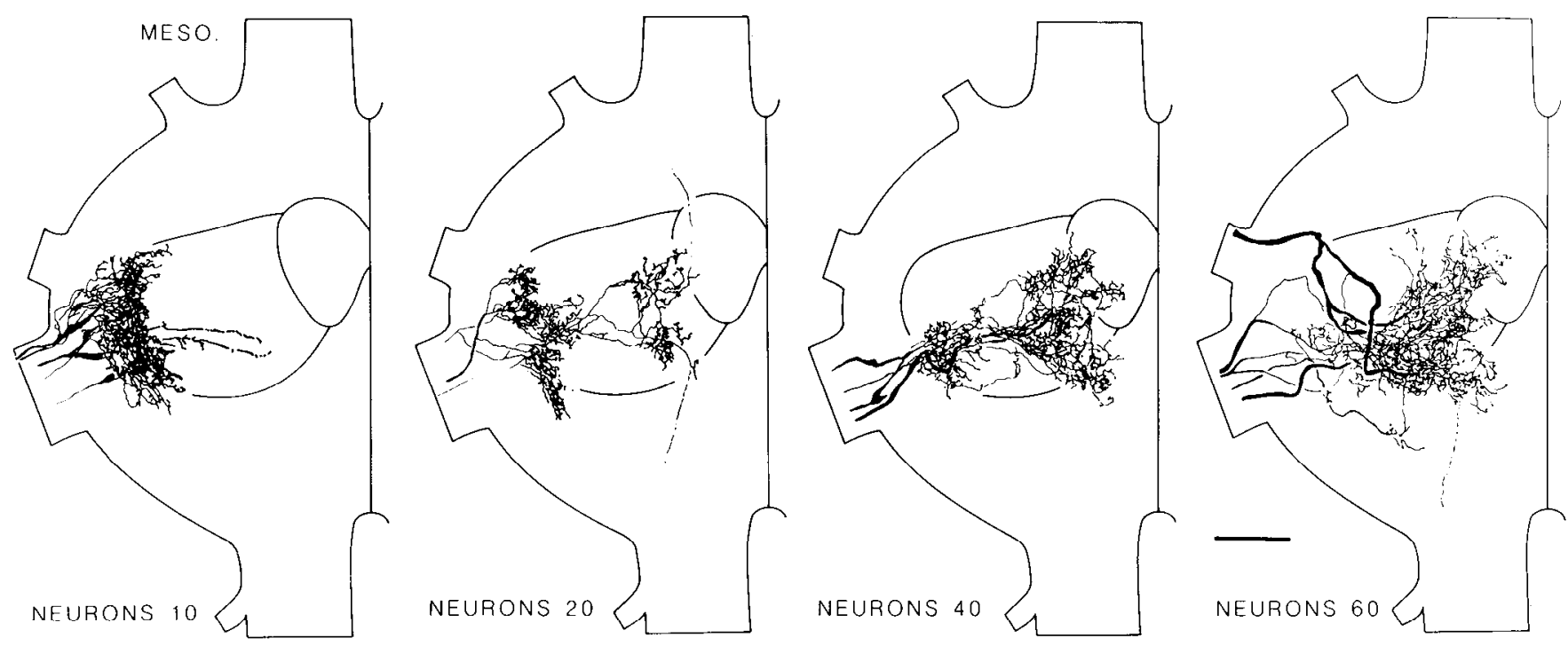

Figure 6. Similarities and differences among the arborizations of neurons at different positions on the cercus. Neurons of a given identity were standardized for ganglion size and superimposed on a common hemiganglionic outline. The superimposition provides an indication of the variance for each neuron. A different neuron is shown in each panel. Note the gradual medial shift as one examines neurons at successively more medial positions on the cercus. The conventions are as in Figure 3 except that each figure is a hemiganglion. The calibration bar represents $100 \mu \mathrm{m}$ (the average of the calibrations for all of the preparations used in the figure).

that the thoracic ganglia are somatotopically ordered (Altman, 1980; Hustert et al., 1981) just as the terminal ganglion is (Murphey et al., 1980; Bacon and Murphey, 1981; Murphey, 1981).

Clavate neurons at different proximodistal locations along the cercus have arbors whose anterior-posterior extent in the abdominal ganglion is correlated with their peripheral location (Murphey et al., 1980; Murphey, 1981). This distinction seems to be lost in the ectopic location. We were unable to detect consistent differences among neurons in row 10, even though such differences are obvious in the terminal ganglion. Thus, the correlation between cell body position around the circumference of the cercus and terminal arbor position in the ganglion is retained, but the proximodistal correlates are lost (or are so subtle we cannot detect them).

The pro- and metathorax. An important corollary of our working hypothesis is the idea that the features of the neuropil landscape are common to each ganglion. We stained selected ectopic neurons transplanted to leg stumps other than the mesothorax in order to confirm the serially repeated nature of the organization. We stained two cells, neurons 10 and 60, in many pro- and metathoracic ganglia. Examples of the results, shown in Figure 7, prove that, within the constraints of the differences in ganglion shape, the arborizations of a given neuron are constant in shape and location from ganglion to ganglion. To summarize, clavate neurons situated medially on the ectopic cercus (neurons 40 and 60) grow to the CNS and arborize medially in the thoracic ganglion they encounter. In contrast, clavale neurons situated dorsally (neuron 80) or ventrally (neurons 10 and 20) arborize in lateral thoracic neuropil.

The arborization within thoracic neuropil of individual ectopic clavate neurons (Fig. 5, $A$ and $B$ ) bears some resemblance to the normal arborization within the terminal ganglion formed by the same neuron (Fig. 5, $C$ and
$D$ ). In the terminal ganglion, clavate neurons 40 and 60 tend to arborize medially, as they do in the thoracic ganglia, and neurons 10 and 80 tend to arborize laterally in both the thoracic and terminal ganglion. The repeated nature of the afferent projections in each ganglion will be considered in more detail in the "Discussion."

\section{The projection of ectopic filiform hairs}

The ectopic filiform neuron projection in the mesothoracic ganglion. As stated previously, the ectopic cercus on the mesothoracic leg stump has a normal appearance: the filiform hairs were found to be distributed over the entire surface, whereas clavate hairs occurred only in a small proximomedial patch. Where the two hair populations overlap, adjacent filiform and clavate neurons on the ectopic cercus were found to project to identical regions of mesothoracic neuropil. This is illustrated clearly by a medial, proximal, filiform neuron, located within the clavate array. This neuron tends to arborize medially in the mesothoracic ganglion (Fig. $8 B$, medial) like its neighboring clavate neurons 40 and 60 (Fig. 5, $A$ and $B$ ). In addition, filiform hairs situated just dorsal to the clavate array (adjacent to clavate neuron 80 ) or just ventral to the clavate array (adjacent to clavate neuron 10) project predominantly to the same lateral regions of mesothoracic neuropil as their clavate neighbors (Figs. $8 A$, dorsal and $8 B$, ventral; cf. Figs. $5 A$, neuron 10 and $5 B$, neuron 80 ). All of the ectopic ventral filiform hair afferents that were filled with cobalt have a predominantly lateral arborization in the mesothoracic neuropil. However, for dorsal hairs the situation is more complex; that is the dorsal filiform hairs situated near the clavate array have neurons with lateral arborizations as described previously, whereas dorsal hairs situated further from the clavate array have afferents with more medial arborizations. Therefore, both types of arborization are shown for dorsal hairs in Figure $8 A$ (dorsal). The affer- 

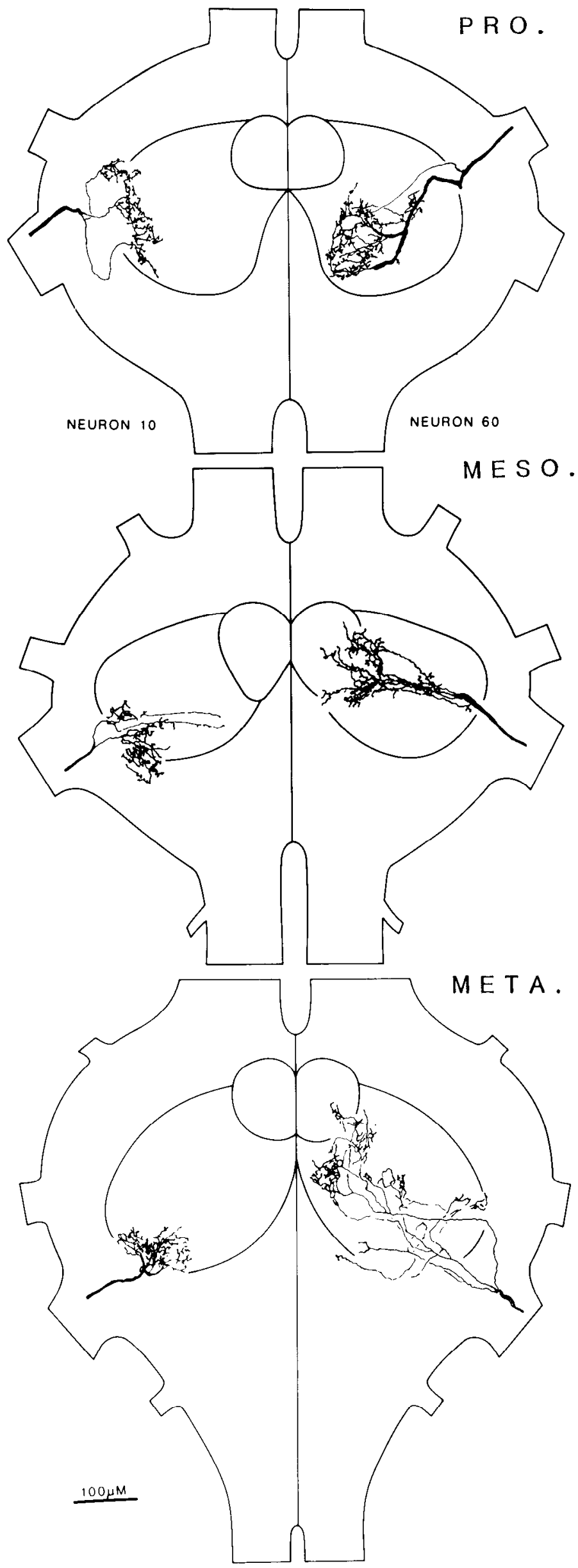

ents from lateral filiform hairs were found to project medially into the mesothoracic ganglion (Fig. 8A, lateral).

To summarize, the ectopic filiform hair afferents project to the same slab of mesothoracic neuropil as the clavate neurons. In more precise terms, neighboring clavale and filiform hairs were shown to have neurons which terminate in the same part of this mechanosensory neuropil. Thus, the somatotopic mapping of cercal surface within the mesothoracic neuropil is the same for both the filiform and clavate systems.

The afferent neurons from filiform hairs, at the same cercal positions as the ectopic filiform afferents investigated, were cobalt filled into the terminal ganglion. Although it is difficult to compare the normal afferent arborizations in the terminal abdominal ganglion with the ectopic ones in the mesothoracic ganglion, a meaningful comparison can be made if one classifies the arborizations as being predominantly medial or lateral. In this fashion, Figure $8 B$ shows, for example, that ventral hair afferents arborize predominantly in lateral neuropil within the terminal and mesothoracic ganglion. Lateral and medial filiform hair afferents tend to arborize medially in the terminal ganglion, as they do in the mesothoracic ganglion (Fig. 8). Dorsal hair afferents arborize laterally or medially within the terminal and in the mesothoracic ganglion: the destination of a particular afferent is a function of a proximity of the hair to the clavate array (Fig. 8). There is some resemblance, therefore, between the arborization formed by particular filiform sensory neurons in their normal target neuropil, in the terminal ganglion, and in the foreign ectopic neuropil of the mesothoracic ganglion.

\section{Anatomy of the target neurons in the mesothoracic ganglia}

Many neurons which are postsynaptic to the cercal afferents in the terminal ganglion are known to pass through the thoracic ganglia (Mendenhall and Murphey, 1974), and ectopic afferents have been reported to synapse with these neurons at these thoracic sites (Edwards and Sahota, 1967; Edwards and Palka, 1976). In the terminal abdominal ganglion there is a correlation between the extent of overlap of sensory neuron terminals with an interneuron's dendrites and the sensory neuron's ability to excite the interneuron (J. P. Bacon and R. K. Murphey, manuscript in preparation). We therefore examined the structure of three giant interneurons in the thorax to determine whether similar anatomical overlap existed at the ectopic sites. The three neurons examined in the thoracic ganglia were the medial giant interneuron (MGI) and the lateral giant interneuron (LGI), which are normally activated by filiform hair afferents (Murphey et al., 1977), and neuron 7-2a, a cell which is normally driven by clavate hairs (Sakaguchi and Murphey, 1983).

Figure 7. A comparison of ectopic neurons arborizing in the three thoracic ganglia. The main point to note is the fact that neuron 10 arborizes primarily laterally in each ganglion, whereas neuron 60 arborizes much more medially in each ganglion. Comparison of these individual arbors with Figure 3 shows that none are outside the range of variability seen in these experiments. Conventions are as in Figure 3. 

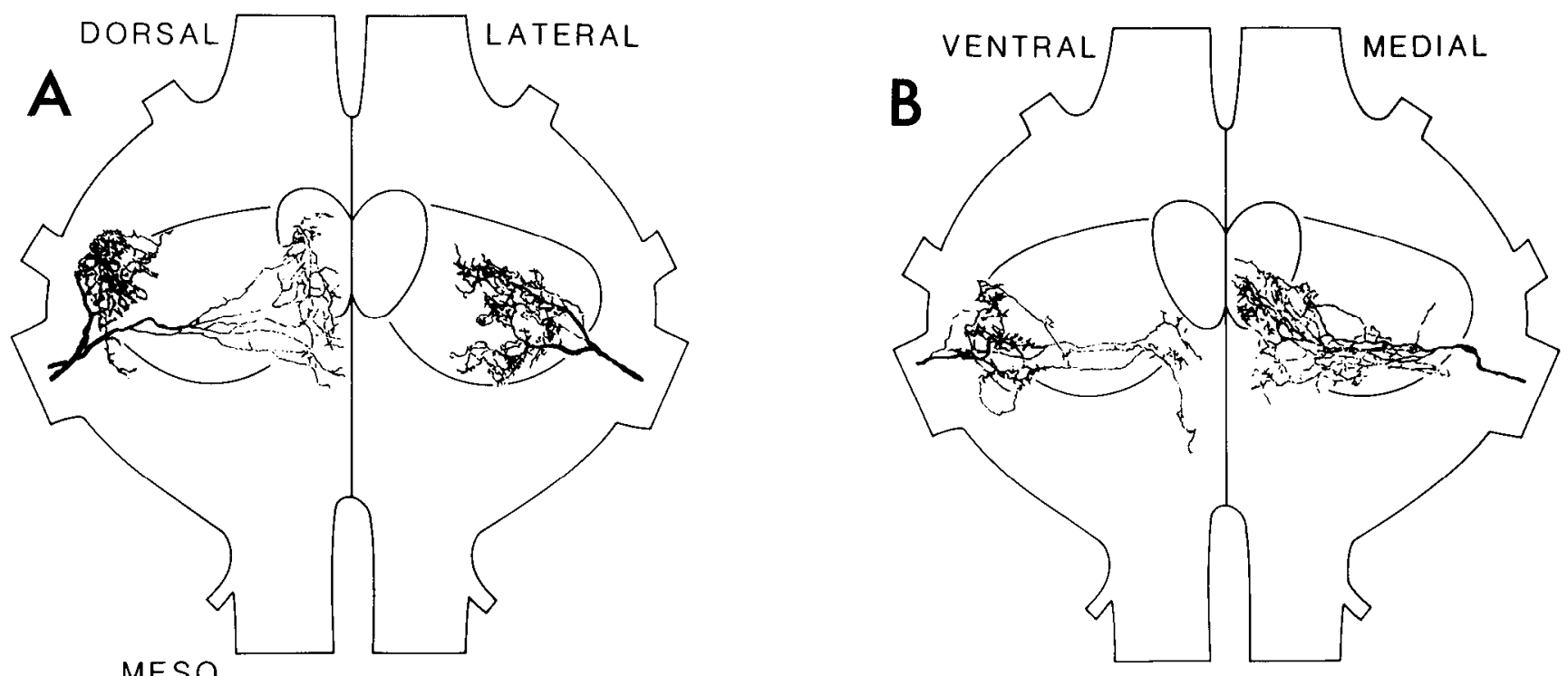

MESO.
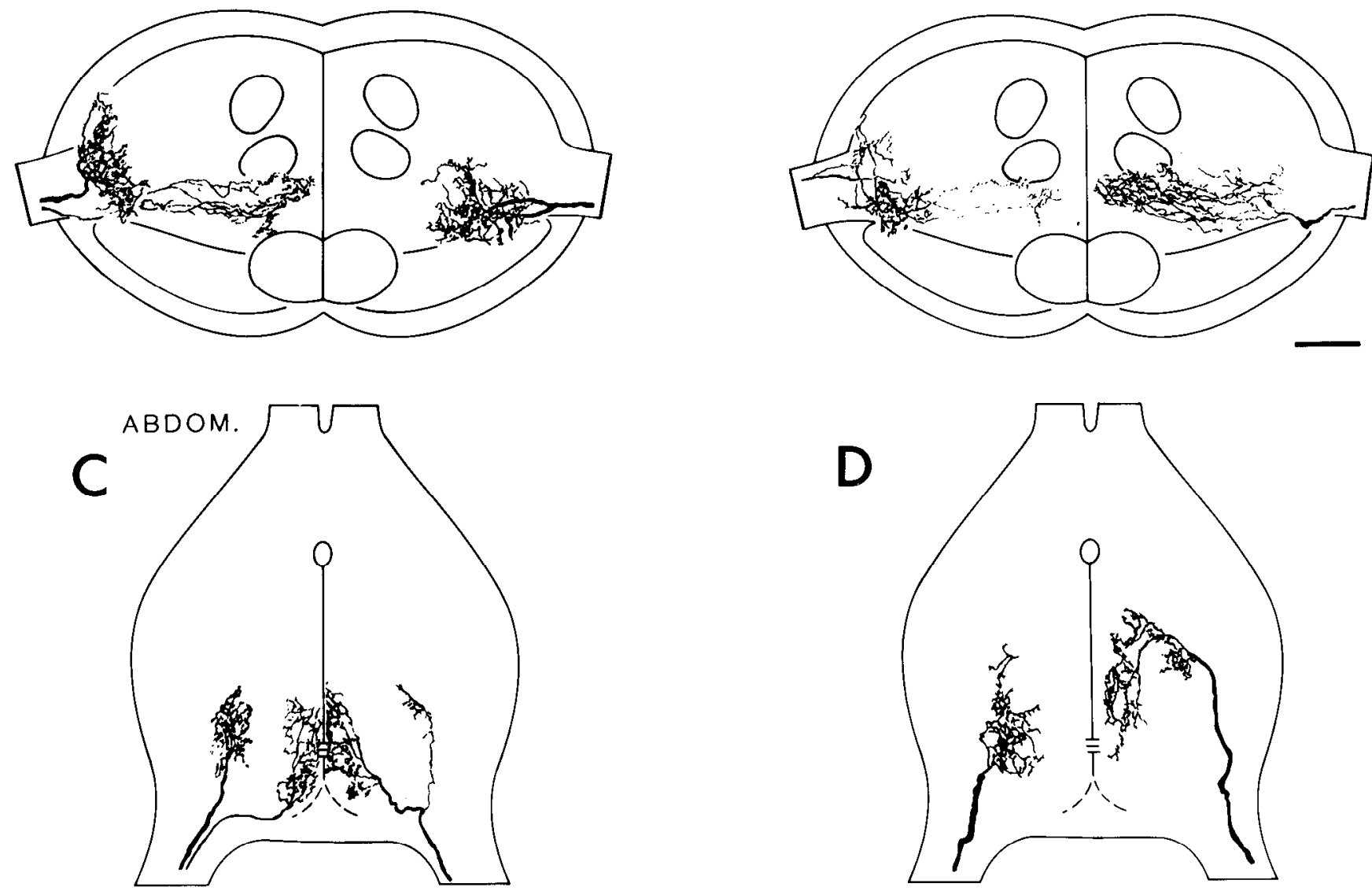

Figure 8. Ectopic filiform hair afferent projections. $A$ and $B$, Upper panels: dorsal views of the afferent arborizations in the mesothoracic ganglion; Lower panels, transverse views of the same arborizations reconstructed from $40-\mu \mathrm{m}$ plastic sections. Neuropilar landmarks are the same as those used in Figure 3. $C$ and $D$, Dorsal views of normal afferent projections to the terminal abdominal 5 th instar ganglion. Scale bars, $100 \mu \mathrm{m}$.

The axons of both the MGI and LGI are found in the VIT in the thoracic ganglia. Both neurons extend a series of short axon collaterals toward the midline in each of the thoracic ganglia (MGI is shown in Fig. 9). The distal ends of these collateral arbors are large bulbous structures (up to $8 \mu \mathrm{m}$ diameter), distinctly larger than the varicosities and spines of these neurons seen in the terminal ganglion, and we suspect that they are presynaptic to thoracic neurons rather than postsynaptic. There is very little overlap between these giant neuron collaterals in the thoracic ganglia and the ectopic afferent projection in the same ganglia. However, if monosynaptic 

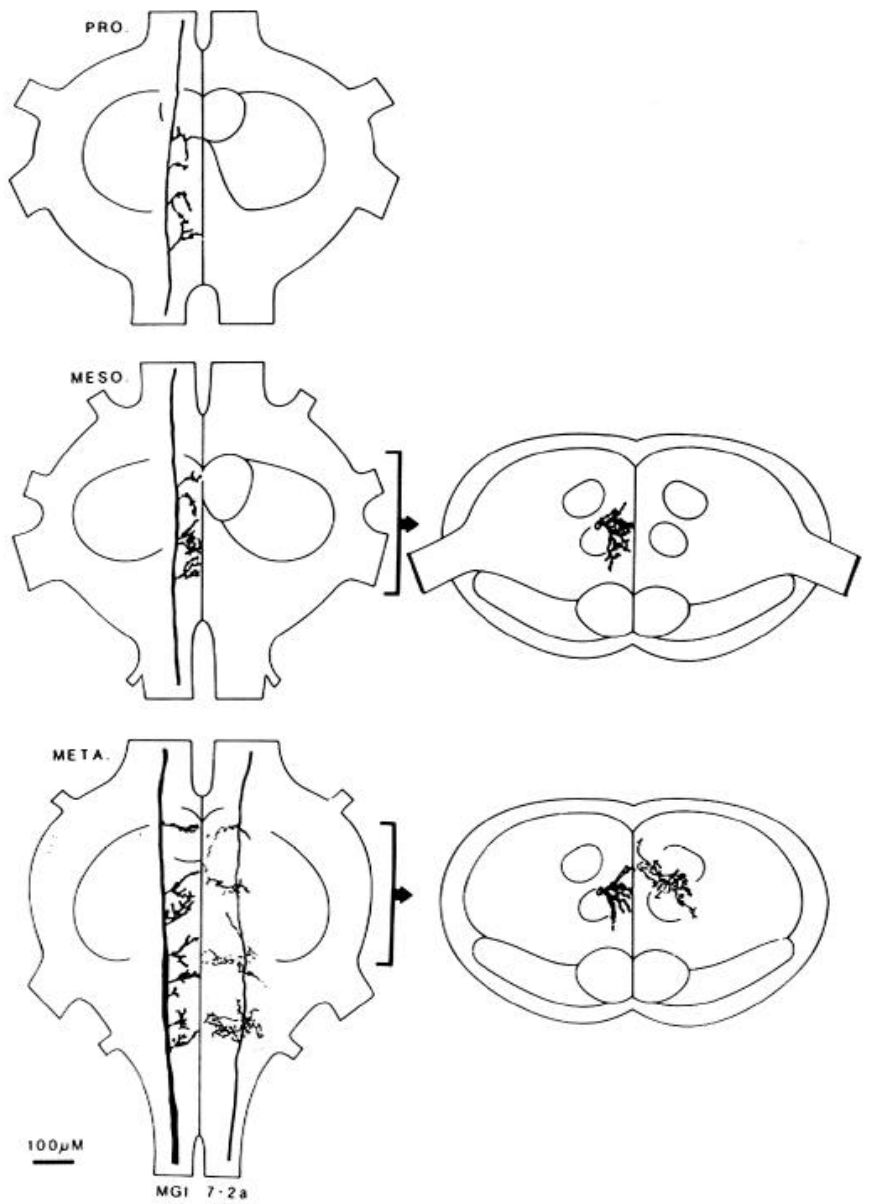

Figure 9. The anatomy of the MGI and interneuron 7-2a in the thoracic ganglia. Both neurons are shown in horizontal (left half of figure) and transverse (right half) view. The brackets indicate that extent of the ganglia used to construct the transverse views. Neuropilar landmarks are as in Figure 3. Neuron 7-2a was filled from an abdominal recording site and filled well only in the metathorax. Although it was stained in the mesothorax, it was too pale to draw accurately. The structure of the LGI (not shown) is very similar to that of MGI, although it has fewer medial-going branches in each of the thoracic ganglia.

contacts are being formed, then medial or lateral afferents are more likely to do so since their afferents tend to project more medially in the ganglion.

Neuron 7-2a has an axon in the dorsal intermediate tract within the metathoracic ganglion. The cell was only well stained in the metathoracic ganglion, although we know that it extends anteriorly at least as far as the prothoracic ganglion. Within the metathorax, the cell possesses numerous fine medial collaterals, distinct in their appearance from the bulbous collaterals of the MGI and LGI. The function of these branches (pre- versus postsynaptic) is unknown and their overlap with the ectopic afferent projection is very small.

\section{Lack of connectivity between ectopic afferents and previously identified interneurons}

A saline-filled electrode placed over the cut end of an insect mechanosensitive hair will record the activity of the bipolar cell beneath it (Bacon and Tyrer, 1979).
Recordings made in this fashion from both the filiform and clavate sensory neurons on the ectopic cercus showed the sensilla to be functional, with action potentials being produced in response to moving the hair with the electrode.

Extracellular recording with wire hook electrodes from the connective between the pro- and mesothoracic ganglia ipsilateral to the ectopic cercus revealed action potentials associated with stimulating the ectopic cercus with sound $(500 \mathrm{~Hz}, 85 \mathrm{~dB})$, wind, or a light touch (Fig. $10 a$ ). The entire animal, except the ectopic cercus, was covered with wax during this experiment. Since activity in response to these stimuli then disappeared on removal of the ectopic cercus, it is clear that the ectopic afferents were exciting these interneurons (Fig. 10b). This confirms Edwards and Sahota's (1967) report that ectopic cerci make functional connections within the CNS.

In order to test the possibility that the MGI or LGI was receiving this ectopic afferent input, we made simultaneous extracellular recordings from the ventral nerve cord near the terminal ganglion and from the connective between the pro- and mesothoracic ganglia. Stimulating the normal cerci with sound produced a volley of ascending action potentials in the abdominal nerve cord (Fig. $10 c$, trace $a b d$.). Triggering the sweep on these large action potentials, which are known to be the activity of the MGI and LGI (Murphey et al., 1977), revealed small time-locked potentials at the thoracic recording site (not shown). The small amplitude of these potentials suggested that neither the MGI nor the LGI could easily be recorded in the thorax and that the larger action potentials in Figure $10 c$ (trace $p-m)$ and in Edwards and Sahota (1967) could not be attributed to the MGI or LGI. The action potentials visible in Figure $10 c($ trace $p-m)$ at the thoracic recording site must be due to the sound stimulation of the ectopic cercus or other thoracic receptors. We then blocked all filiform hair movement on the normal cerci with Vaseline and moved the loudspeaker to optimally stimulate the ectopic cercus and again examined interneuronal responses. Action potentials in response to a tone were readily detected at the thoracic recording site (Fig. 10d, trace $p-m$ ) but no descending MGI or LGI action potentials were produced at the abdominal recording site (Fig. 10d, trace abd.). (The small action potentials recorded in the abdomen during this part of the experiment were the activity of an unidentified unit.) These results suggest that sensilla on the ectopic cercus provide no suprathreshold input to the MGI or LGI in the thoracic ganglia.

In order to search for any subthreshold input to the MGI or LGI from sensilla on the ectopic cercus, we recorded intracellularly from these neurons in the mesothoracic ganglion. Good impalements of the axons were associated with a resting potential of -40 to $-50 \mathrm{mV}$ and overshooting action potentials in response to stimulation of the normal, posterior cerci. These action potentials were associated one-to-one with those recorded extracellularly in the abdominal nerve cord. No subthreshold synaptic activity was seen in response to stimulation of the ectopic cercus with sound, wind, or a gentle touch (Fig. 10e). We conclude that ectopic filiform hair afferents do not synapse with the LGI or the MGI in the mesothoracic ganglion. 
a.

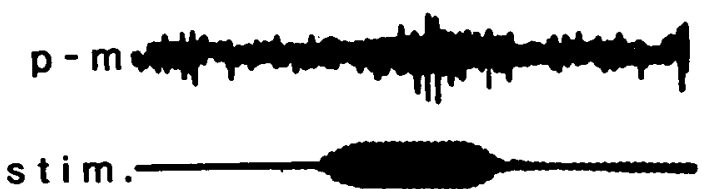

b.
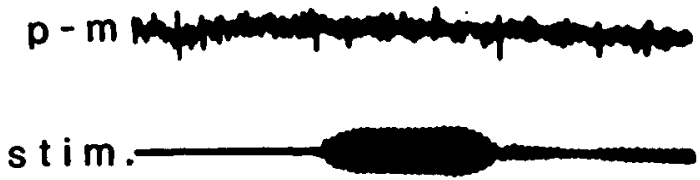

C.

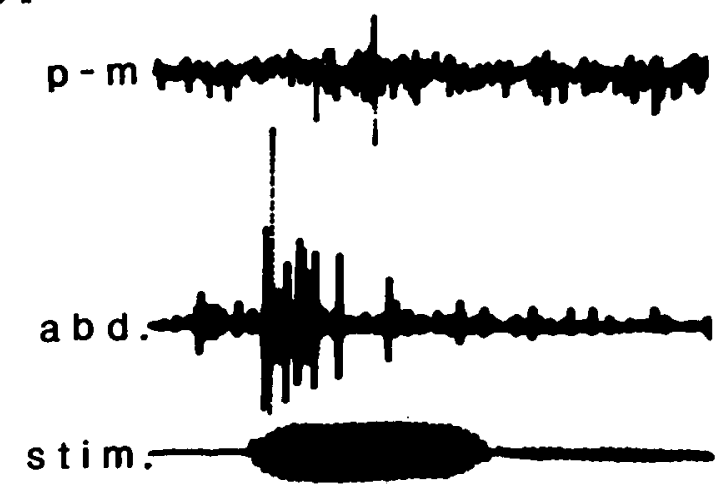

d.

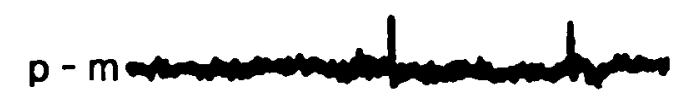

\section{a b}

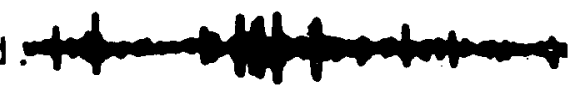

s t i m

Figure 10. Electrophysiological investigation of putative target interneurons of the ectopic cerci. $a$, Action potentials recorded extracellularly from the pro-mesothoracic connective $(p-m$, top trace) in response to sound stimulation $(500 \mathrm{~Hz}, 80 \mathrm{~dB}$; stim., bottom trace) of the ectopic cercus. $b$, No extracellular spikes are seen in response to sound stimulation after removal of the ectopic cercus. The same animal as in $a$ was used. $c$, Sound stimulation of the normal posterior cerci causes a burst of spikes in the MGI and LGI recorded in the abdominal connective ( $a b d$., middle trace). Action potentials in these units are too small to be seen at the thoracic recording site; those visible are due to stimulation of the ectopic cercus. $d$, Covering the posterior cerci and moving the loudspeaker to optimally stimulate the ectopic cercus results in the stimulation of an unidentified unit in the thorax, but no MGI or LGI spikes are visible at the abdominal recording site. The origin of the small spikes seen in the abdominal recording in response to sound stimulation is unknown, although it is clearly not the activity of the MGI or LGI. The same animal as in $c$ was used. The time scale for $a$ to $d$ is $20 \mathrm{msec}$. $e$, Three consecutive traces of an MGI recorded intracellularly in the mesothoracic ganglion ( $M G I$, top trace) and extracellularly in the abdominal connective (abd., middle trace). The traces are triggered by a delayed sound stimulus (stim., bottom trace) placed to optimally stimulate the ectopic cercus. In one of the three traces, three spikes are produced by stimulation of the posterior cerci, the delay from abdomen to mesothorax being approximately $2 \mathrm{msec}$. No subthreshold synaptic potentials are seen in MGI as a result of the sound stimulation. The vertical scale for the intracellular trace is $1 \mathrm{mV}$; the Horizontal scale is $5 \mathrm{msec}$. $f$, Receptive fields from the right position-sensitive interneuron (7-2a) following selective deletion of clavate hairs on the normal abdominal cerci and ectopic cercus (ectopic cercus was transplanted to the right mesothorax). The receptive fields $(a, b$, and $c)$ are polar coordinate plots of the responses from the right interneuron 7 $2 \mathrm{a}$ to $60^{\circ}$ tilt displacements at $22.5^{\circ}$ increments around the yaw axis. Each datum point represents the mean frequency from four trials in the same preparation. The strippled region at the center denotes the base level action potential activity at the normal, primary orientation. Along the longitudinal or pitch axis, $0^{\circ}$ corresponds to pitch forward and $180^{\circ}$ to pitch backward. Along the transverse or roll axis, $90^{\circ}$ corresponds to roll right and $270^{\circ}$ to roll left. The control receptive field, a, was obtained with all clavate hairs present on both the normal cerci and ectopic cercus. Receptive field $b$ was obtained 45 min following the removal of all clavate hairs on the normal cerci, thus leaving intact only clavate hairs on the ectopic cercus in the right mesothorax. Receptive field $c$ was then obtained following the removal of the remaining clavate hairs on the ectopic cercus. 
We next examined the clavate equilibrium system for a similar assessment of connections between ectopic clavate receptors and one of their normal targets, interneuron 7-2a. The animal was mounted on a tilt table and the neural activity was monitored in response to changes in its orientation (experimental details are provided in Sakaguchi and Murphey, 1983). We first characterized the interneuron receptive fields after immobilizing all of the filiform hairs, leaving only clavate hairs free to move on both the normal abdominal cerci and the ectopic cercus on the right mesothoracic coxa. The receptive fields for both left (not shown) and right (Fig. 10f) 7-2a were normal in this situation. (Since both left and right 7-2a receptive fields were normal, we will consider only the right 7-2a which, based on its anatomy, is the obvious candidate to receive ectopic innervation.) All clavate hairs on the normal, right cercus were then deleted, the specimen was allowed to recover from the trauma ( 45 $\mathrm{min}$ ), and the receptive field was again mapped. Following the removal of clavate receptors on the normal, right cercus, thus leaving only clavate hairs on the ectopic cercus, the action potential activity in neuron $7-2 \mathrm{a}$ was no longer modulated by displacements with respect to the gravity field. Furthermore, removal of the clavate hairs from the ectopic cercus had no additional effect on its activity (Fig. 10f). Based on the extracellular axonal recordings, there is no evidence that the ectopic clavate afferents are synapsing with their normal targel, neuron 7-2a. If the ectopic afferents have synapsed with neuron $7-2 \mathrm{a}$, it is such a weak connection that it cannot influence the neuron's axonal activity. This neuron is too small to reliably impale in the thorax and therefore we were unable to examine any subthreshold ectopic inputs to the neuron.

By recording in the ipsilateral connective near the anterior end of the mesothoracic ganglion with hexami-

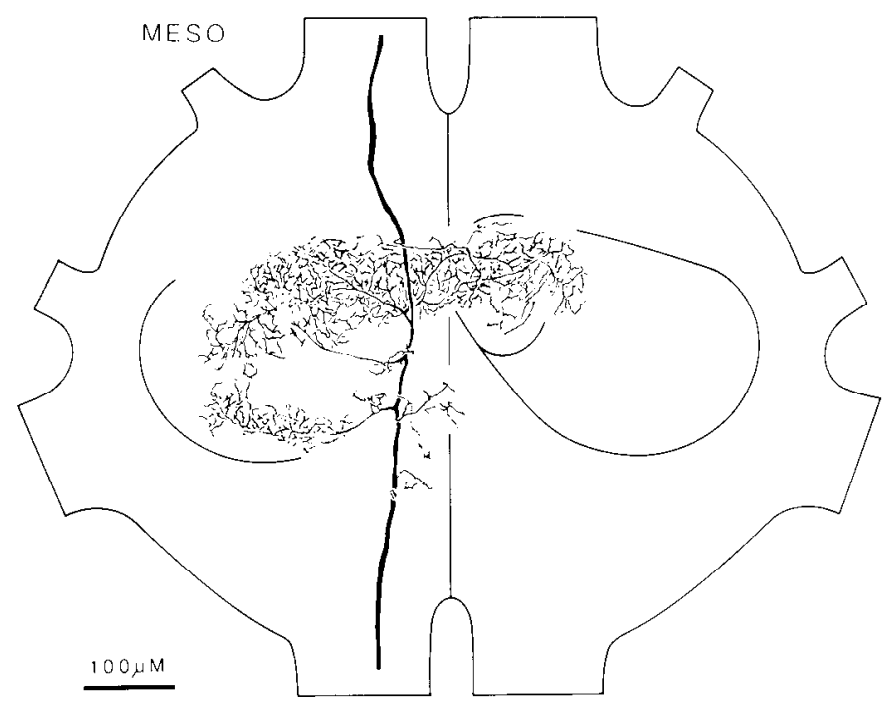

Figure 11. Horizontal view of the anatomy of an interneuron in the mesothoracic ganglion which responded to gentle brushing of the ectopic cercus. The cell's axon is found in the VIT and it has dense arborizations in the ventral neuropil occupied by the ectopic arborization (cf. Fig. 3). The cell has an almost identical branching pattern in each of three thoracic ganglia. Neuropilar landmarks are as in Figure 3. necobaltic chloride-filled microelectrodes, we attempted to stain and characterize those interneurons (recorded extracellularly in Fig. 10a) which receive input from the ectopic cercus. Several units have been encountered and stained. They responded to wind stimulation or a light touch to the ectopic cercus. We have not yet found units that respond to sound stimulation. The structure of a neuron in the mesothoracic ganglion which responded vigorously to gentle brushing of the ectopic cercus is shown in Figure 11. The axon is situated in the VIT and the cell has a dense, ventral arborization, parts of which are situated in the vicinity of the ectopic cercal projection. This cell has a very similar structure in the pro- and metathoracic ganglia and we presume it receives afferent input in each ganglion. The level of staining of this neuron was sufficient to show that its cell body is not situated in any of the thoracic ganglia and that the cell extends at least as far anteriorly as the subesophageal ganglion and posteriorly as far as the first free abdominal ganglion. A more detailed description of interneurons receiving ectopic cercal input is in preparation and we hope to establish a relationship between the position of an interneuron's dendritic branching within the ectopic arborizations and the afferent input it receives.

\section{Discussion}

\section{Synaptogenesis in ectopic locations}

Our experiments have reexamined the pioneering work of Edwards and Sahota (1967) and confirm their finding of functional synaptic connections between the ingrowing ectopic afferent neurons and interneurons in the CNS. One of the oft quoted results of their work is the conclusion that afferents recognize their target neurons wherever they encounter them (e.g., Edwards and Palka, 1976; Bate, 1978; Anderson et al., 1980; Anderson, 1981). However, in the present study we have demonstrated that ectopic cercal afferents do not synapse with the abdominal giant neurons (MGI or I.GI) in the thorax, even though there is some anatomical overlap. Thus we suggest that ectopic afferents cannot recognize the giant neurons (MGI or LGI) in the mesothorax. Consistent with this idea, the structure of the thoracic arborizations of these giant neurons is quite distinct from that of their dendrites in the terminal ganglion. The varicosities are very large (up to $8 \mu \mathrm{m}$ diameter) and few in number and we suspect that they are primarily output areas of the neuron. Ectopic afferents did make synapses with other interneurons accessible to them in the mesothorax (Fig. 11), demonstrating that they can make functional synapses. We assume it was these unusual largets that were recorded by Edwards and Sahota (1967) and that these cells normally receive mechanoreceptive input in the thoracic ganglia.

Anderson (1981) has investigated a similar experimental paradigm in the locust. She showed that patches of head hairs, transplanted to the thorax, made no functional connections with the tritocerebral commissure giant (TCG) wind-sensitive interneuron, one of their normal targets in the brain (Bacon and Tyrer, 1978). In this case, no anatomical overlap between ectopic afferents and the TCG occurred; thus cell-cell recognition could not be tested. 


\section{The basic structure of the arthropod segmental} ganglion

The neuropil of the insect segmental ganglion has classically been thought of as a sandwich with motor areas dorsal, sensory areas ventral, and association areas in the center. The advent of dye injection techniques and the resultant explosion of information concerned with single-neuron anatomy has caused some revision of this classical view. For example, these new techniques have demonstrated that sensory afferents project to virtually all regions of neuropil (see Altman, 1980, for a review). This does not mean that the sensory projection is random because we can see that sensory neuropil is partilioned in two major ways.

First, as we have shown here, afferents from the ectopic cercus innervate two major regions of thoracic neuropil. The mechanosensory clavate and filiform hair afferents project to a slab of neuropil in the middle of the ganglion, which stretches from the midline to the lateral edge (Fig. $3 B$ ), whereas afferents of the appressed hairs (of unknown function) arborize in a more ventral slab of neuropil (R. K. Murphey, unpublished observation). This separation of the two areas of neuropil in thoracic ganglia is not an artifact of the experimental surgery inasmuch as leg afferents are known to selectively innervate one or the other of the areas outlined in Figure 3 (Hustert, 1978; Eibl and Huber, 1979; Bräunig et al., 1981; Hustert et al., 1981; Pflüger et al., 1981; D. Wohlers, personal communication). Thus, based on the destination of their afferents within neuropil, there seem to be two main types of afferent termination from the insect appendage.

Second, within these areas of neuropil, the terminals form a topographic projection of the body surface. Normally, cercal mechanoreceptor neurons with neighboring cell bodies in the periphery arborize in neighboring regions of the cercal glomerulus (Murphey et al., 1980; Murphey, 1981; Bacon and Murphey, 1981; J. P. Bacon and R. K. Murphey, manuscript in preparation) and it was these near-neighbor relations that we looked for in experimental situations. We found that these near-neighbor relations were preserved when ectopic sensory neurons arborized in foreign ganglia; for example, clavate neurons 40 and 60 and medial filiform hairs all arborize medially in their target area. Clavate and filiform neurons at other locations on the ectopic cercus arborize in other regions of this neuropil. Thus as Altman (1980) had speculated, this area of neuropil appears to contain “. . . a spatially organized map of the body surface. ..."

An important result of the present work is the demonstration that a topologically similar ordering of cercal afferents occurs in each of the ganglia studied. This is readily detected in the three thoracic ganglia (Fig. 7) but is less obvious if one tries to compare thoracic and abdominal projections. A difficulty is that the terminal abdominal ganglion is distorted relative to the more primitive shape of the thoracic ganglia. It is located at the terminus of the CNS where nerve locations are highly modified compared with the basic pattern. The main modification in nerve trunks is the stretching of major nerves posteriorly with respect to the ganglion. Thus afferent axons enter the terminal ganglion at its posterior end. In contrast, in the mesothoracic ganglion ectopic afferents enter nearer the lateral edge (in nerve 3 or 5). In spite of these differences in ganglion shape and nerve trunk location, parallels between abdominal and thoracic projections can be detected. Those afferents which project to a medial area of the cercal glomerulus (e.g., clavate neuron 60 , and medial and lateral filiform hairs, Figs. $5 B$ and $8, A$ and $B$ ) also arborize most medially in the mesothorax, whereas those afferents which project more laterally in the cercal glomerulus (e.g., clavate neuron 10 or ventral filiform hairs) possess terminal arborizations confined to lateral regions of the mesothoracic ganglion. We presume that these are homologous areas of neuropil in the various ganglia. These results suggest that circumferential lociation of a sensory neuron on the leg will be correlated with arbor location in the thoracic ganglion. Specifically, we would predict that anterior and posterior surfaces of the leg will be represented more medially in neuropil and dorsal and ventral surfaces will be represented more laterally.

Anderson (1981) has also demonstrated the serially repeated nature of segmenlal ganglia by transplanting locust head hair epidermis to the pro-, meso-, and metathorax. She found that head hair afferents that normally cross the ganglionic midline did so at ectopic sites, and those that normally remained ipsilateral remained ipsilateral in ectopic ganglia. Thus, based on our results and those of Anderson (1981), it seems reasonable to conclude that the features of the neuropil landscape used by growing afferents to organize the cercal sensory projection in the terminal ganglion are present in each segmental ganglion. What these repeated features of the ganglionic neuropil are remains a mystery, but they seem likely to be associated with the dendritic surfaces of the target neurons which form the scaffolding of the ganglia even before sensory afferents arrive in the CNS (Edwards and Chen, 1979; Shankland and Goodman, 1982).

One aspect of the normal abdominal projection seems to be lost in the ectopic situation. In the terminal ganglion, neurons at a common circumferential position on the cercus but with different proximodistal positions vary in the anterior-posterior extent of their terminal arbors (Murphey et al., 1980). We were unable to detect a similar order in foreign ganglia. We suspect that the anteriorpostcrior extent of the terminal arborizations can only be expressed if the sensory cells reach adjacent ganglia. The pro- and mesothoracic, though not the metathoracic ganglia are derived from a single segment, whereas the terminal abdominal ganglion is derived from five embryonic ganglia (Panov, 1966; Matsuda, 1976). In Drosophila, sensory neurons associated with mesothoracic macrochaetes invade adjacent ganglia of the fused nervous system to a greater or lesser extent as a function of their anterior-posterior position on the mesothorax (Ghysen, 1980). The cercal glomerulus is thought to be derived from more than one segment (J. S. Edwards, unpublished observation; G. Jacobs, unpublished observation) and mechanoreceptors may therefore span more than one segment. Thus, in the terminal ganglion, the proximodistal axis of the cercus may be mapped across segment boundaries, whereas there is little or no opportunity to express this property in thoracic ganglia. An alternative possibility is that our techniques do not have enough resolution to detect such differences. 
In summary, the results reveal a partitioning of neuropil based on the type of the receptor. Within the various projection areas, a further partitioning based on topography of the receptors at the body surface is seen. Such a plan has important implications for first order synaptic connections because we would expect the location of the dendritic field of any motor or interneuron to be correlated with its synaptic input from sensory neurons.

\section{The role of somatotopy in determining synaptic connectivity}

The idea that somatotopy might determine neuronal connectivity is an old one in neurobiology. It is implicit in much of the work in the field that the overlap of a known afferent projection with the dendrites of a postsynaptic cell is a mechanism in itself for determining orderly connections. However, the limits of this type of structure-function correlation have not been tested. It remains to be seen which aspects of synaptic connectivity are determined by the topography of the target cell with respect to the afferent projection and which aspects are determined by other mechanisms. For example, one can conceive of specific chemoaffinities which could determine a neuron's ability to synapse with a specific follower cell. If all neurons in an array were so specified with respect to a target array, then somatotopic order might emerge as the result, and not the determinant, of the connectivity. In order to test the relevance of somatotopy, one must perturb the order in one or both sets of neurons. The rationale is that if somatotopy is a mechanism in itself for producing ordered synaptic connections, then in a pertrubed system connectivity will be altered in a predictable manner.

This problem has been approached at two levels. At the macro level, the hypothesis is that the gross anatomical location of an interneuron or motor neuron's dendrites determines which inputs are available to it. At the micro level, the shape of a neuron's dendritic field with respect to the afferent projection is presumed to determine the shape of the receptive field (Young, 1960; Colonnier, 1964; Tieman and Hirsch, 1982). Our experiments address this issue at both levels of analysis by perturbing the afferent projection. At the macro level, the transplantation of an abdominal appendage to the thorax reveals a lack of affinity of the afferents for their normal targets, an affinity which seems to function only in the abdominal areas. At the micro level, the identification of neurons which do receive ectopic input allows us to search for a correspondence between afferent topography, dendritic topography, and receptive field characteristics of these foreign targets.

In summary, our experiments suggest a number of conclusions about neurospecificity in the insect nervous system. First, the abdominal giant neurons are probably recognizable to afferents only in the terminal ganglion and lack the molecules required to receive synapses outside the terminal abdominal ganglion. Second, the features which guide the growth of a given afferent under normal conditions are serially repeated in each ganglion and can be interpreted by ectopic afferents. Third, the high fidelity of the projection within the thoracic ganglia suggests a mechanism for ordering the synaptic connec- tivity between afferents and first order neurons throughout the animal.

\section{References}

Altman, J. S. (1980) Functional organisation of insect ganglia. In Advances in Physiological Science. Vol. 23: Neurobiology of Invertebrates, J. Salanki, ed., pp. 537-555, Pergamon Press, Oxford.

Anderson, H. (1981) Projections from sensory neurons developing at ectopic sites in insects. J. Embryol. Exp. Morphol. (Suppl.) 65: 209-224.

Anderson, H., J. S. Edwards, and J. Palka (1980) Developmental neurobiology in invertebrates. Annu. Rev. Neurosci. 3: 97-140.

Bacon, J. P., and R. K. Murphey (1981) Cricket cercal filiform hairs have a somatotopic projection in the terminal ganglion. Soc. Neurosci. Abstr. 7: 4.

Bacon, J., and N. M. Tyrer (1978) The tritocerebral commissure giant (TCG): A bimodal interneurone in the locust, Schistocera gregaria. J. Comp. Physiol. 126: 317-325.

Bacon, J., and N. M. Tyrer (1979) The innervation of the windsensitive head hairs of the locust, Schistocera gregaria. Physiol. Entomol. 4: 301-309.

Bate, C. M. (1978) Development of sensory systems in arthropods. In Handbook of Sensory Physiology, M. Jacobson, ed., Vol. 9, pp. 2-53, Springer-Verlag, New York.

Bräunig, P., R. Hustert, and H. J. Pflüger (1981) Distribution and specific central projections of mechanoreceptors in the thorax and proximal leg joints of locusts. I. Morphology, location and innervation of internal proprioceptors of proand metathorax and their central projections. Cell Tissue Res. 216: 57-78.

Brogan, R. T., and R. M. Pitman (1981) Axonal regeneration in an identified insect motoneurone. J. Physiol (Lond.) 319: 34P-35P.

Colonnier, M. L. (1964) The tangential organization of the visual cortex. J. Anat. 98: 327-344.

Edwards, J. S., and S. W. Chen (1979) Embryonic development of an insect sensory system: The abdominal cerci of Acheta domesticus. Wilhelm Roux's Arch. Dev. Biol. 186: 151-178.

Edwards, J. S., and J. Palka (1976) Neural generation and regeneration in insects. In Simpler Networks and Behavior, J. C. Fentress, ed., pp 167-185, Sinauer Associates, Inc., Sunderland, MA.

Edwards, J., and T. S. Sahota (1967) Regeneration of a sensory system: The formation of central connections by normal and transplanted cerci of the house cricket Acheta domesticus. J. Fxp. 7ool. 166: 387-395.

Eibl, E., and F. Huber (1979) Central projections of tibial sensory fibers within the three thoracic ganglia of crickets (G. campestris L., G. bimaculatus De Geer). Zoomorphologie 92: 1-17.

French, V. I., P. J. Bryant, and S. V. Bryant (1976) Pattern regulation in epimorphic fields. Science 193: 969-981.

Ghysen, A. (1980) The projection of sensory neurons in the central nervous system of Drosophila: Choice of the appropriate pathway. Dev. Biol. 78: 521-541.

Hustert, R. (1978) Segmental and interganglionic projections from primary fibres of insect mechanoreceptors. Cell Tissue Res. 194: 337-351.

Hustert, R., H. J. Pflüger, and P. Bräunig (1981) Distribution and specific central projections of mechanoreceptors in the thorax and proximal leg joints of locusts. III. The external mechanoreceptors: The campaniform sensilla. Cell Tissue Res. 219: 97-112.

Matsuda, R. (1976) Morphology and evolution of the insect abdomen: With special reference to developmental patterns and their bearing upon systematics. Pergamon Press, New York. 
Matsumoto, S. G., and R. K. Murphey (1977) The cercus-togiant interneuron system of crickets. IV. Patterns of connectivity between receptors and the medial giant interneuron. $J$. Comp. Physiol. 119: 319-330.

Mendenhall, B., and R. K. Murphey (1974) The morphology of cricket giant interneurons. J. Neurobiol. 5: 565-580.

Mollenhauer, H. H. (1964) Plastic embedding mixtures for use in electron microscopy. Stain Technol. 39: 111-114.

Murphey, R. K. (1981) The structure and development of a somatotopic map in crickets: The cercal afferent projection. Dev. Biol. 88: 236-246.

Murphey, R. K., J. Palka, and R. Hustert (1977) The cercus-togiant interneuron system of crickets. II. Response characteristics of two giant interneurons. J. Comp. Physiol. 119: 285300.

Murphey, R. K., A. Jacklet, and L. Schuster (1980) A topographic map of sensory cell terminal arborizations in the cricket CNS: Correlation with birthday and position in a sensory array. J. Comp. Neurol. 191: 53-64.

Panov, A. (1966) Correlations in the ontogenetic development of the central nervous system in the house cricket Gryllus domesticus and the mole cricket Gryllotalpa gryllotalpa $L$. (Orthoptera, Grylloidea). Entomol. Rev. 45: 179-185.

Pflüger, H. J., P. Bräunig, and R. Hustert (1981) Distribution and specific central projections of mechanoreceptors in the thorax and proximal leg joints of locusts. II. The external mechanoreceptors: Hair plates and tactile hairs. Cell Tissue Res. 216: 79-96.

Pipa, R. L., and E. F. Cook (1959) Studies on the hexapod nervous system. I. The peripheral distribution of the thoracic nerves of the adult cockroach, Periplaneta americana (L). Ann. Entomol. Soc. Am. 52: 695-710.

Sakaguchi, D. S., and R. K. Murphey (1983) The equilibrium detecting system of the cricket: Physiology and morphology of an identified interneuron. J. Comp. Physiol., in press.

Shankland, M., and C. S. Goodman (1982) Development of the dendritic branching pattern of the medial giant interneuron in the grasshopper embryo. Dev. Biol. 92: 489-506.

Tieman, S. B., and H. V. B. Hirsch (1982) Exposure to lines of only one orientation modifies dendritic morphology of cells in the visual cortex of the cat. J. Comp. Neurol. 211(4): 353362.

Tyrer, N. M., and G. E. Gregory (1982) A guide to the neuroanatomy of locust suboesophageal and thoracic ganglia. Philos. Trans. R. Soc. Lond. (Biol.) 297: 91-124.

Young, J. Z. (1960) The visual system of the octopus. I. Regularities in the optic lobes of octopus in relation to form discrimination. Nature 186: 836-844. 